\title{
The Accuracy of Cardiovascular Pooled Cohort Risk Estimates in U.S. Older Adults
}

\author{
Michael G. Nanna, MD D, Eric D. Peterson, MD, MPH, Daniel Wojdyla, MS, and \\ Ann Marie Navar, MD, PhD
}

Duke Clinical Research Institute, Duke University School of Medicine, Durham, NC, USA.

\begin{abstract}
BACKGROUND: The ACC/AHA guidelines for primary prevention rely on the Pooled Cohort Risk Equations (PCE) risk estimates of atherosclerotic cardiovascular disease (ASCVD) to guide treatment decisions. In light of the PCE being derived in younger populations, their accuracy in older adults is uncertain.

OBJECTIVE: To evaluate the predictive accuracy and calibration of the PCE in older individuals.

DESIGN AND SETTING: We estimated CVD predicted and observed risk among individuals from four large prospective cohort studies: Cardiovascular Health Study, Multiethnic Study of Atherosclerosis, Framingham Original, and Framingham Offspring.
\end{abstract}

PARTICIPANTS: 12,527 overall individuals without ASCVD, including 9864 individuals aged 40-74 years and 2663 aged $\geq 75$ years.

MEASUREMENTS: We examined the operating characteristics of the PCE to estimate 5-year risk of stroke, MI, and CHD death overall and by age and sex strata. The associations between individual components of the PCE and cardiovascular events by age group ( $\geq 75$ vs 40 74 years) were also evaluated.

RESULTS: The PCE had low discrimination for 5-year ASCVD risk in older ( $\geq 75$ years) (c-statistic $=0.62,95 \%$ CI 0.60-0.65) vs. younger (40-74 years) adults (c-statistic $=0.75,95 \%$ CI 0.73-0.76). Calibration of the PCE was suboptimal in both older and younger adults, overestimating risk in the highest risk groups. Performance of the PCE in older adults was similarly poor when stratified by sex and age $\geq 80$ years.

LIMITATIONS: Since the PCE were derived from similar cohorts, though using different age groups and exams, this analysis likely overestimates the performance of the PCE.

CONCLUSION: The performance of the PCE for ASCVD risk estimation in older adults is suboptimal; new models to effectively risk-stratify older adults are needed.

KEY WORDS: older adults; geriatric; risk; atherosclerotic cardiovascular disease; pooled cohort equations.

$\mathrm{J}$ Gen Intern Med 35(6):1701-8

DOI: $10.1007 / \mathrm{s} 11606-019-05361-4$

(c) Society of General Internal Medicine 2019

Electronic supplementary material The online version of this article (https://doi.org/10.1007/s11606-019-05361-4) contains supplementary material, which is available to authorized users.

Received July 24, 2019

Revised July 24, 2019

Accepted September 12, 2019

Published online October 30, 2019
T here are more than 20 million Americans $\geq 75$ years old, Cardiovascular disease (CVD) remains the number one cause of death among older Americans. ${ }^{2}$ The current American College of Cardiology.

(ACC)/American Heart Association (AHA) guidelines rely on the Pooled Cohort Risk Equations (PCE) as the main tool for estimating primary prevention treatment thresholds. Nevertheless, the PCE were developed based on a middle-aged population (40-79 years, mean age 56 years $)^{3}$ and no studies have been dedicated to comparing their use specifically among older adults $\geq 75$ years old. Furthermore, a number of studies have suggested that the PCE may overestimate CVD risk in general, but particularly in females. 4,5

We used data from the National Heart, Lung, and Blood Institute's Pooled Cohorts (Cardiovascular Health Study [CHS]-Visit 3, Multi-Ethnic Study of Atherosclerosis [MESA]-Exam 1, Framingham Original Exam 24, and Framingham Offspring Exam 7) to: 1) compare the performance of the PCE for predicting cardiovascular risk (ischemic stroke, myocardial infarction [MI], and cardiovascular death) in older adults ( $\geq 75$ years old) with younger adults (40-74 years old); 2) investigate whether truncating age at 79 years rather than using chronologic age in the equation improved performance of the PCE in older adults ( $\geq 75$ years); 3 ) assess performance of the PCE for predicting cardiovascular risk in individuals $\geq 80$ years old, females $\geq 75$ years old, and males $\geq 75$ years old; and 4) evaluate whether the association between the individual risk factors included in the PCE and CVD events differed between younger (40-74 years old) and older ( $\geq 75$ years) patients.

\section{METHODS}

\section{Data Description}

The original PCE were developed using data from the Atherosclerosis Risk in Communities Study (ARIC), the Coronary Artery Risk Development in Young Adults Study (CARDIA), CHS (baseline or year 2 exam), Framingham original (visit cycle 11), and Framingham Offspring (Visit cycles 1 and 3, where the participant could be included twice if event free at visit 3). We included PCE data from four more recent followup exams that included patients over the age of 75 years old 
and had at least 5-years of follow-up: 1) CHS Visit 3 (19921993); 2) MESA Exam 1 (2000-2002); 3) Framingham Original Exam 24 (1995-1998); and 4) Framingham Offspring Exam 7 (1998-2001). These datasets were selected to utilize the most contemporary data available while still maintaining a minimum of 5 years of follow-up and containing the information needed to calculate the PCE. ${ }^{6}$ Inclusion criteria for the cohorts ( $\geq 75$ vs. $40-74$ years old) were patients who had $\geq 5$ years follow-up and no history of established atherosclerotic cardiovascular disease (ASCVD) at the time of enrollment, with a known status for common risk factors including hyperlipidemia, hypertension, diabetes, and smoking ( $\geq 75$ years old, $N=2667 ; 40-74$ years old, $N=9865$ ). Missingness in the final population was minimal with four patients missing systolic blood pressure (SBP) and/or diastolic blood pressure (DBP) in the $\geq 75$ year-old cohort (final analysis population $N=2663$ ) and one patient missing high-density lipoprotein cholesterol (HDL-C) in the 40-74 year-old cohort (final analysis population $N=9864$ ).

\section{Covariates}

We included the following baseline characteristics in the population of older adults: age, sex, race, body mass index, blood pressure, diabetes, smoking, medications, lipids levels, and creatinine clearance. Variables included for calculation of the PCE include gender, age, race, SBP, anti-hypertensive treatment, diabetes, smoking, total cholesterol (mg/dL), and HDL$\mathrm{C}(\mathrm{mg} / \mathrm{dL})$.

\section{Outcome(S)}

The ASCVD PCE 5-year risk calculation has been previously described $^{7}$ and was used in the original validation of the PCE. ${ }^{8}$ The equation predicts "hard" ASCVD risk, meaning the risk of first occurrence of nonfatal MI, stroke (including fatal and non-fatal), and coronary heart disease (CHD) death. For that reason, our primary outcome of interest was a real-world composite of CHD death, fatal and non-fatal MI, and fatal and non-fatal stroke at 5 years compared to the event rate predicted by the PCE.

\section{Statistical Analysis}

Baseline characteristics of participants 40-74 years old vs. $\geq 75$ years were summarized for continuous variables with median and quartiles, and compared using the Wilcoxon test. Categorical variables were summarized as frequencies and percentages, and compared using chi-square test. For each participant, the predicted probability of having an MI, stroke, or CHD death event within the first 5 years of follow-up was computed using the ACC/AHA PCE. We used the KaplanMeier method to compute the observed probability of having an event within the first 5 years of follow-up with $95 \%$ confidence intervals (CIs) for individuals $\geq 75$ years overall, stratifying by sex. The analyses for the overall population were carried out two ways: 1) using actual chronologic age for individuals $>79$ years (for whom the equation was not previously validated); and 2) truncating at age 79 by substituting age 79 for those $\geq 80$ years. Performance of the PCE was also tested in the $80+$ year-old subgroup. In addition, we calculated cumulative incidence rates in order to account for the competing risk of non-cardiovascular death and compared those absolute event rates to the Kaplan-Meier estimates and PCE predicted rates.

For comparison with younger adults, we also assessed discrimination and calibration of the PCE for individuals 4074 years old from the same cohorts at the same exams. Calibration was assessed using $p$ values comparing observed and expected rates based on the Greenwood-Nam-D'Agostino calibration test ${ }^{9}$, and discrimination assessed using the Cstatistic.

To better understand variability of risk predictors between older and younger adults, we examined the association between each factor in the PCE and CVD risk by age group. For each of these factors, we report the unadjusted and adjusted hazard ratio (HR) with $95 \% \mathrm{CI}$ and univariate c-index, stratified by younger (40-74 years) vs. older ( $\geq 75$ years) adults. Adjusted HRs for each risk factor were compared between the two age groups by testing interactions in a Cox regression model including main effects for all risk factors and interactions of each risk factor with age group.

\section{RESULTS}

\section{PCE Performance in Older Adults}

We evaluated 2663 adults aged $>75$ years in the pooled cohorts $(59 \%$ female; median age 78 , interquartile range [IQR] 76-82) and 9884 adults aged 40-74 years (56\% female; median age 62, IQR 54-70) without any pre-existing history of ASCVD. Baseline characteristics by age strata are provided in Table 1. Older patients ( $\geq 75$ years) were more frequently white $(76.6 \%$ vs. $64.8 \%, p<0.0001)$, with lower body mass index (median 26.0 vs. $27.5, \mathrm{p}<0.0001$ ), higher blood pressure (median SBP $137 \mathrm{mmHg}$ vs. $124 \mathrm{mmHg}, p<0.0001$ ), more diabetes $(12.4 \%$ vs. $10.6 \%, p=0.008)$, lower estimated glomerular filtration rate (53.1 vs. $80.8, p<0.0001)$, and were less frequently on lipid-lowering medications $(11.5 \%$ vs. $14.0 \%, p=0.0009$ ).

The PCE were less able to discriminate patients with vs. without 5 -year CVD events among older adults $\geq 75$ years old (c-statistic $=0.62,95 \%$ CI $0.60-0.65$; Fig. 1A) compared with improved discrimination among younger patients from the same cohorts (c-statistic $=0.75,95 \%$ CI $0.73-0.76$; Fig. 1B). Poor calibration in both populations $(\geq 75$ years: Chi square $=$ $60.37, \mathrm{p}<0.0001 ; 40-74$ years: Chi-square $=55.36, \mathrm{p}<$ 0.0001 ) was driven by overestimation of risk in the highest risk individuals. Overestimation of risk by the PCE among patients $\geq 75$ years old was even more pronounced when the competing risk of non-cardiovascular death was accounted for 
Table 1. Baseline characteristics by age group.

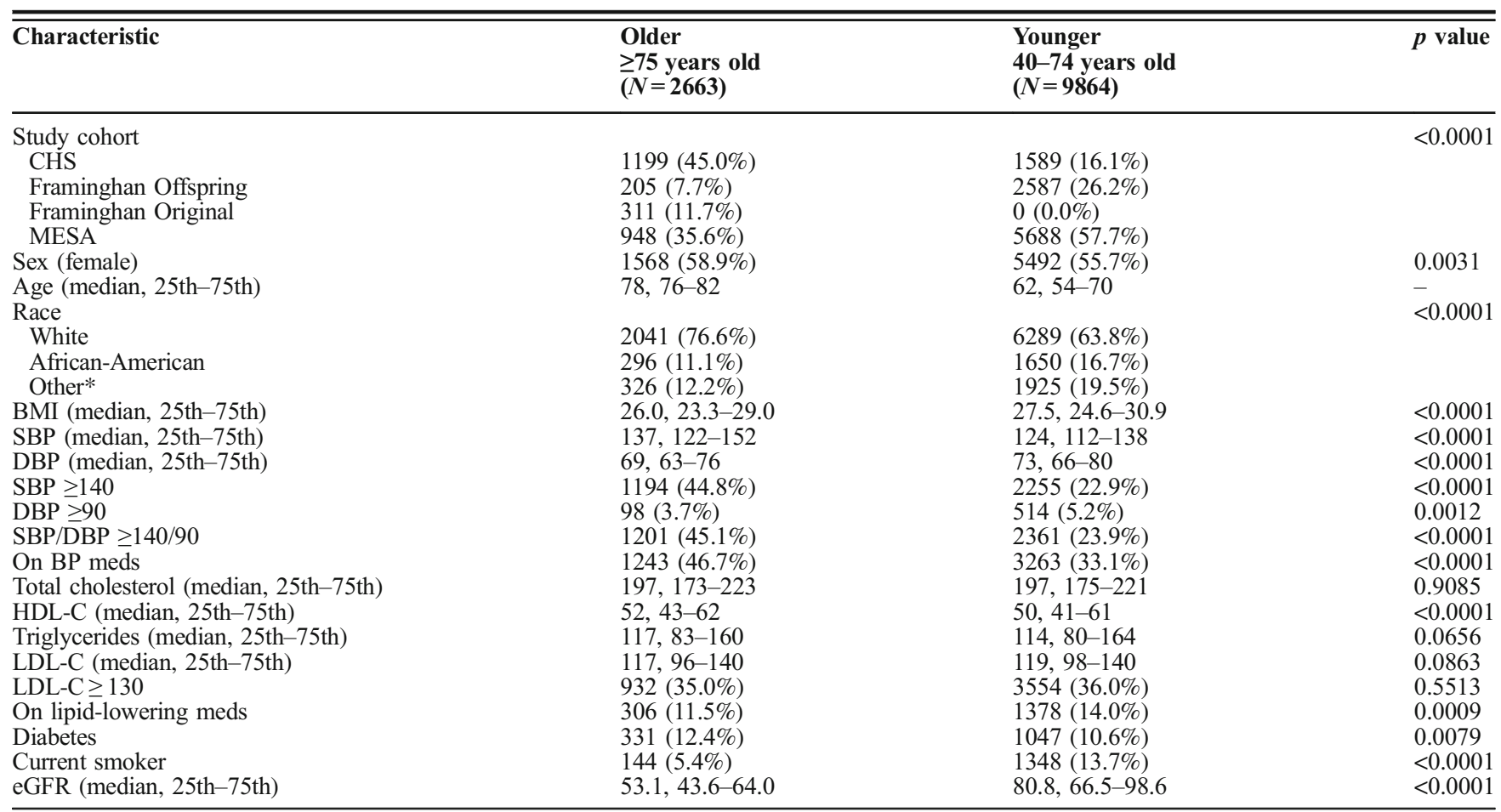

BMI, body mass index; CHS, Cardiovascular Health Study; DBP, diastolic blood pressure; HDL-C, high-density lipoprotein cholesterol; LDL-C, lowdensity lipoprotein cholesterol; MESA, Multi-Ethnic Study of Atherosclerosis; SBP, systolic blood pressure

*Includes Asian and other non-White or Black races.

(eTable 1). When age was truncated at 79 years for the population overall (i.e., substituting age 79 for individuals $\geq 80$ years), discrimination remained poor (c-statistic $=0.61$, 95\% CI 0.59-0.63), though calibration of the PCE improved (Chi-square $=9.84, p=0.36$; eFigure 1$)$.

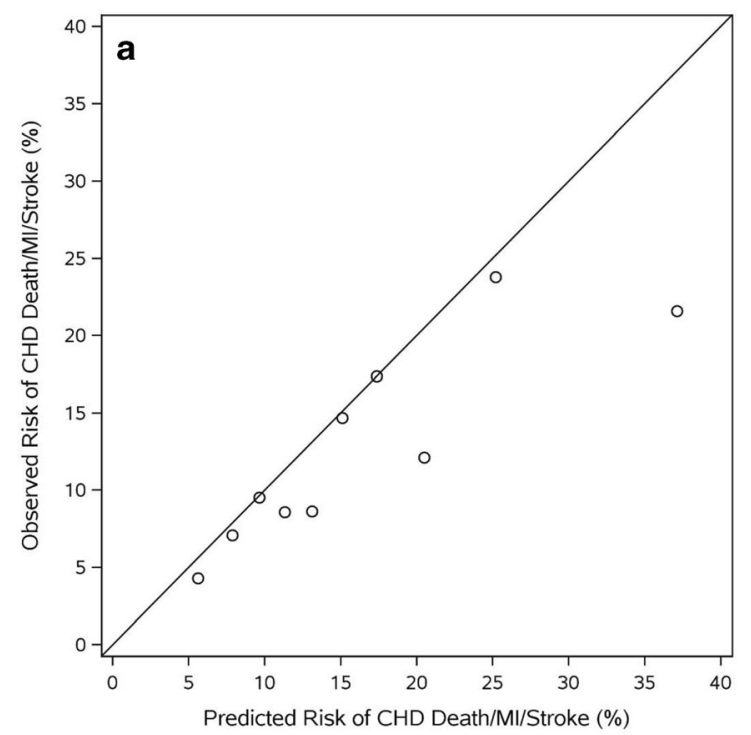

When the PCE was applied to only those aged $\geq 80$ years $(N=904)$, both discrimination (c-statistic 0.60 , 95\% CI $0.56-$ 0.63 ) and calibration (Chi-square $=38.0, p<0.0001$ ) remained poor (eFig. 2). Risk-overestimation was again driven by over-estimation of risk in the highest risk subjects with

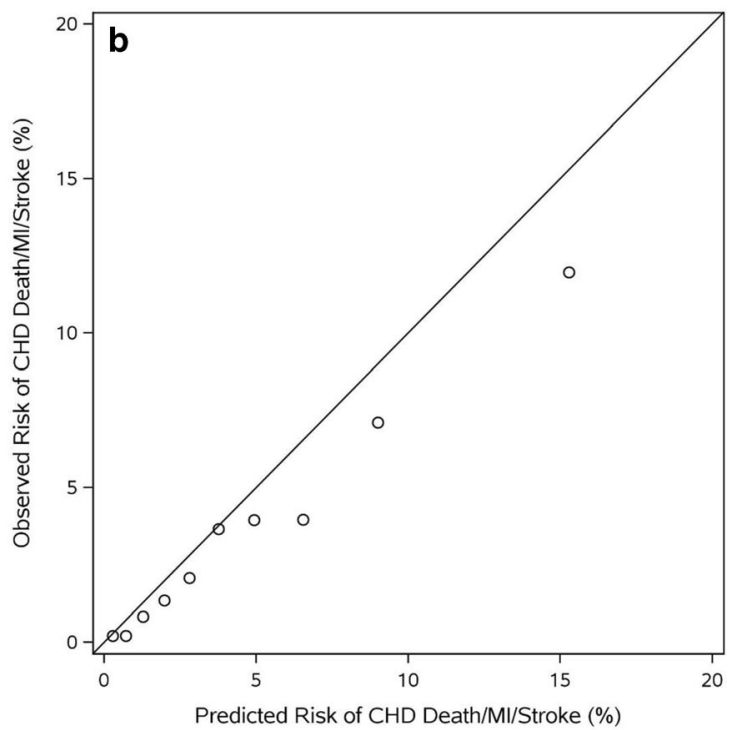

Fig. 1 A. Risk Prediction among Older Adults ( $\geq 75$ years): Calibration of the Pooled Cohort Equations This figure demonstrates the performance of the PCE among older adults ( $\geq 75$ years old). Calculations are made using the patients chronologic age (including patients $>79$ years). Calibration chi-square: $60.37, p<0.0001$ CHD, coronary heart disease; MI, myocardial infarction; PCE, Pooled Cohort Risk Equations. B. Risk Prediction among U.S. Adults (Age 40-74 Years): Calibration of the Pooled Cohort Equations This figure demonstrates the calibration of the PCE among adults (40-74 years old). Calibration chi-square: 55.36, $p<0.0001$ CHD, coronary heart disease; MI, myocardial infarction; PCE, Pooled Cohort Risk Equations; U.S., United States. 
better alignment among those at intermediate risk. PCE performance was similarly sub-optimal among older females and males (Figure 2), with comparable discrimination (females: cstatistic $=0.62,95 \%$ CI $0.59-0.65$; males: c-statistic $=0.64$, 95\% CI 0.61-0.68) and calibration (females: Chi-square $=$ 25.1, $p=0.003$; males: Chi-square $=54.2, P<0.0001)$.

\section{Predictive Power of PCE Risk Factors in Young Vs. Old}

We evaluated the association between the individual risk factors in the PCE and CVD events in younger (40-74) vs. older ( $\geq 75$ years) patients (Table 2 ). Female sex becomes less protective in older adults (adjusted HR 0.85, CI 0.72-1.01) compared with younger adults (adjusted HR 0.61, CI 0.53 0.71) ( $p$ value for interaction 0.004), as does HDL-C ( $\mathrm{p}$ value for interaction 0.01). Smoking status is less strongly associated with cardiovascular events in older adults (adjusted HR 1.53, CI 1.11-2.10) compared with younger adults (adjusted HR 2.38 , CI 2.00-2.82) ( $\mathrm{p}$ value for interaction $=0.02$ ). The adjusted association between CVD events and age, race, total cholesterol, SBP, blood pressure medications, and diabetes remains similar between younger and older patients.

\section{DISCUSSION}

Older adults are a rapidly growing demographic in the United States. Although current guidelines for CVD prevention focus on adults 40-74 years old, primary prevention of ASCVD will become increasingly important in older adults as life expectancies increase. Unfortunately, we found that a key tool used in younger adults to guide preventive therapy is inadequate for use in older populations. The PCE, which is the most commonly used ASCVD risk prediction model in the United States, demonstrated low decimation and poor calibration when applied to older adults ( $\geq 75$ years), compared with younger patients (40-74 years). In particular, the models tended to overestimate risk among those at highest-risk thresholds and suboptimal performance overall appears to be partially driven by the increasing competing risk of noncardiovascular mortality in older adults. The model's low performance did not vary when applied to either men or women or when the sample was limited to those aged $>80$ years. These findings highlight the need for age-specific risk models to estimate CVD risk in older adults.

Our study is among the first to evaluate the performance of the PCE for predicting cardiovascular risk (ischemic stroke, MI, and CVD death), specifically among older adults ( $\geq 75$ years old), demonstrating that the PCE perform poorly in this population. Given the well-described sex differences in cardiovascular risk factors and CVD prevalence, ${ }^{10,}{ }^{11}$ we evaluated the discrimination and calibration of the PCE in women vs. men $\geq 75$ years old. Yet unlike what has been shown for younger adults, ${ }^{4}$ we did not observe sex differences in the overall performance of the PCE, with relatively poor performance among both sexes. Similar to how the PCE tends to overestimate risk in the younger populations for which it was derived, ${ }^{7}, 12-16$ the PCE overestimated risk in high-risk older adults in our study. Overestimating risk in high-risk individuals who are already likely to meet a treatment threshold is not necessarily a compelling reason to avoid using this widely available risk calculator in older adults. A recent study by Saeed et al. evaluated an older cohort of subjects with 4 years of follow-up who participated in the Atherosclerosis Risk in Communities (ARIC) study. ${ }^{17}$ The investigators found that the PCE actually underestimated risk in the highest risk
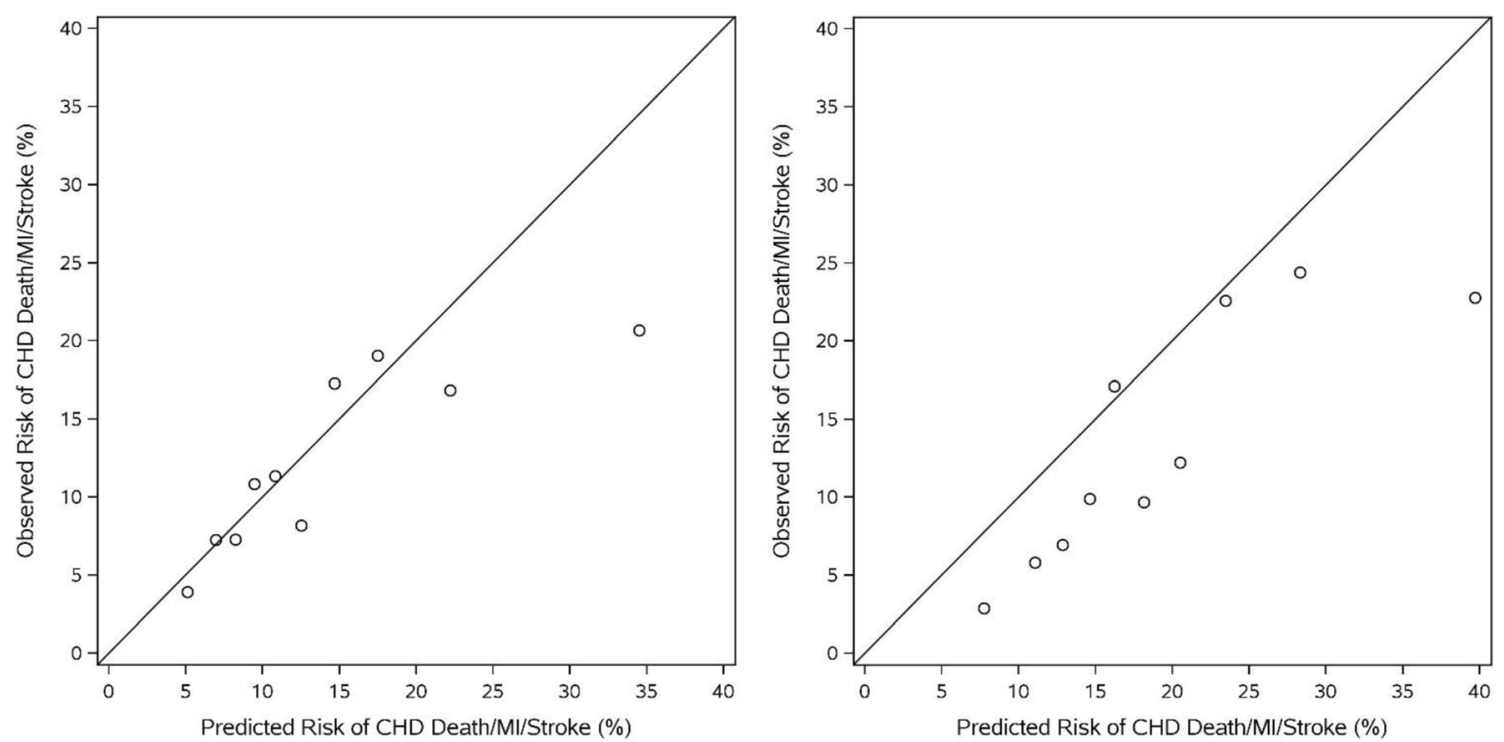

Figure 2. Calibration Plots for Women vs. Men $\geq 75$ Years Old This figure demonstrates the calibration of the PCE among women vs. men $\geq 75$ years old. Females: Calibration chi-square: $25.06, p=0.0029$ Males: c-index: Calibration chi-square: $54.15, p<0.0001$ CHD, coronary heart disease; MI, myocardial infarction; PCE, Pooled Cohort Risk Equations. 


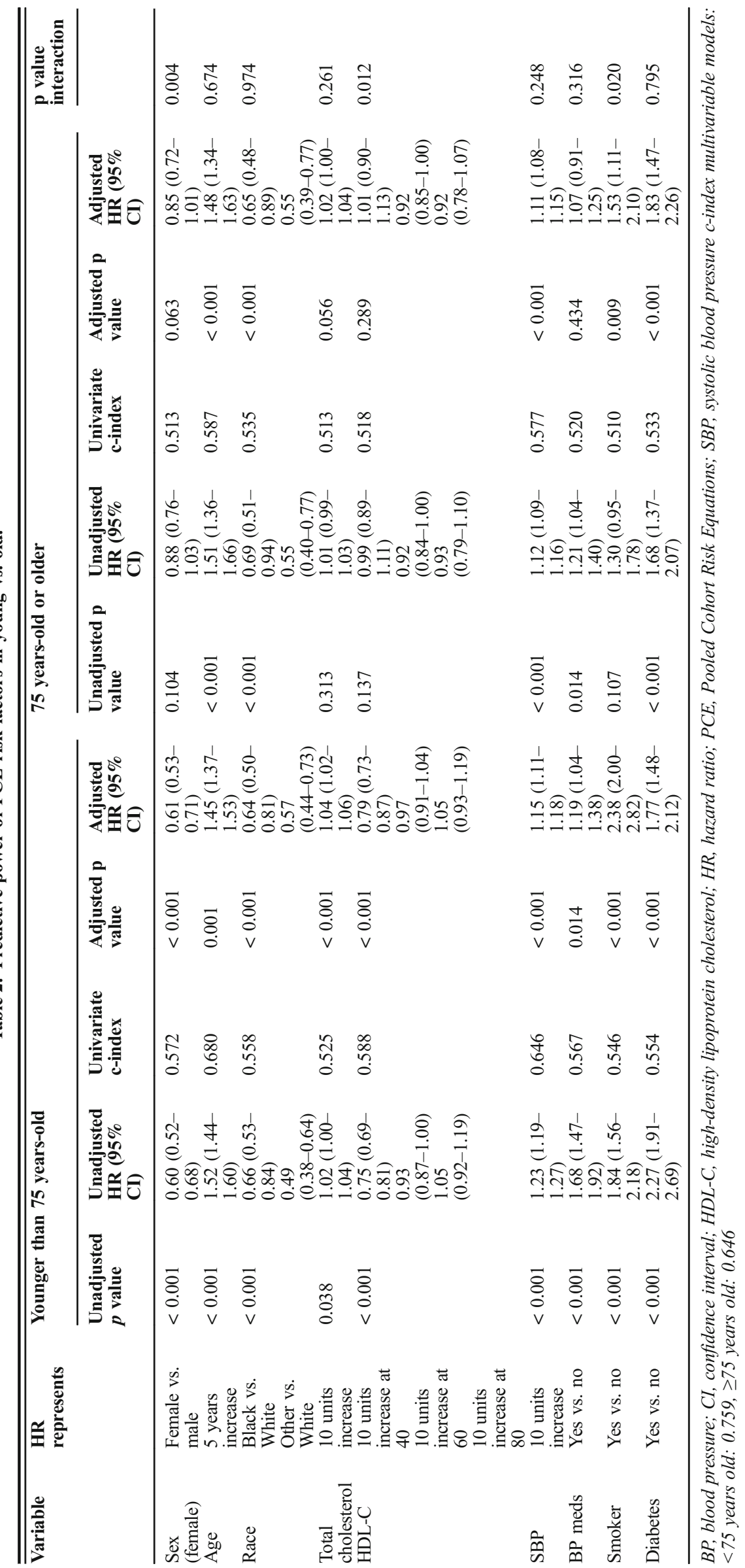


patients, identifying that $52 \%$ of all CVD events occurred in individuals in the upper two deciles of risk, further motivating treatment consideration of these high-risk groups.

A strength of our analysis is the robust size (>900) of the cohort of individuals $\geq 80$ years old. Octogenarians are living longer and may benefit from risk-reducing therapies, but evidence supporting these therapies are limited, ${ }^{1,18}$ which can lead to treatment differences in older adults at high-risk for future cardiovascular events. ${ }^{19}$ Given increasing life expectancies, many patients ages 75 and older can expect to live well over a decade. A clearer understanding of future cardiovascular risk in this population can inform patient-centered conversations regarding pharmacologic therapies with limited randomized-controlled evidence. This is in line with the most recent cholesterol guideline recommendation to consider statin discontinuation (IIb) among older adults where there are concerns around functional decline, multimorbidity, frailty, or reduced life expectancy limiting the potential benefits of statin therapy. ${ }^{20}$ Accurate cardiovascular risk stratification is a necessary but not sufficient first step to precede a broader riskbenefit discussion that accounts for these various other factors that should be considered when making shared prevention treatment decisions in this population. For example, if a patient cannot afford their medication, lacks a support system to reliably take their medications, have limited life expectancy due to other comorbidities, or simply have priorities of care that do not align with aggressive risk reduction, their cardiovascular risk may take on lesser priority in these decisions. Future models generated for older adults that incorporate cause-specific CVD risks as well as models that account for the competing risk of non-CV death may play an important role in patient shared decision making around CVD prevention.

Accurate cardiovascular risk stratification in this age group can be a challenging due to the lack of validation of the commonly used risk stratification tools in this population. The MESA risk score, which incorporates traditional risk factors and coronary artery calcium score, is a 10-year CHD risk prediction tool derived in patients up to age 84 (mean age in the MESA cohort was $62.1+10.2$ years). ${ }^{21}$ Nevertheless, the MESA risk score was validated in two younger validation cohorts not including individuals $>75$ years (Heinz Nixdorf Recall Study were $45-75$ years old with mean age $59.8 \pm 7.7$ years $^{22}$; Dallas Heart Study subjects were 45 65 years old with mean age $52.7 \pm 5.5$ years $^{23}$ ). Furthermore, the MESA risk score is not widely used by providers and not recommended for use by the current guidelines. The ACC/ AHA PCE ASCVD risk calculator is the current risk estimation tool of choice and recommended by current guidelines, ${ }^{20}$ but this risk tool uses age as a primary driver of risk. Older patients reach a primary prevention treatment consideration threshold of $7.5 \%$ or higher by age 75 regardless of other risk factors. ${ }^{24}$ While clinicians commonly substitute age 79 for patients $\geq 80$ years old when calculating risk in the community setting (most existing calculators do not allow for higher values), our results support using patients' actual chronologic age when calculating their ASCVD risk using the PCE because of slightly better discrimination.

Given the poor performance of the PCE in older adults that have been identified, future geriatric-specific risk stratification tools must be developed and validated in order to improve clinicians' ability to accurately estimate risk. We attempted to identify risk factors included in the PCE that explain the variable predictive value when applied to older adults. For example, we observed that female sex was not nearly as protective for individuals $\geq 75$ years old compared with younger patients; this may be due to a loss of protective hormonal effects following menopause. ${ }^{25}$ The weakened association between smoking status and CVD events among older adults reflects the likely increase in death from non-cardiovascular causes such as malignancy. Non-cardiovascular death, from conditions such as cancer and Alzheimer's disease, is the most common driver of mortality in this population, further emphasizing the balance of cardiovascular risk reduction with the multitude of competing factors that may influence treatment decisions in this population. Because the PCE do not take into account death from other causes, they tended to over-predict cardiovascular risk in older adults. Although the 30-year CVD risk tool developed from the Framingham Offspring cohort by Pencina et al. ${ }^{26}$ did incorporate competing risk into their risk estimates, a shorter-duration model that incorporates competing risk is not available. However, while accounting for competing risk is important to generate a well-calibrated $\operatorname{model}^{27}$, caution should be taken in the clinical application of a model that accounts for the competing risk of non-CV death as competing risk may vary across populations and many of the risk factors that increase risk for non-cardiovascular mortality also increase risk of $\mathrm{CV}$ mortality. For example, someone with a longstanding smoking history may be at high risk for both CVD and malignancy. In a model without competing risk, their CVD risk would be high. Using a model that accounted for their competing risks of non-CV mortality would paradoxically decrease their predicted risk of CVD. Whether it would be appropriate to avoid CVD prevention because a particular patient may die of something else first should be part of a patient and provider conversation, and not driven by the specifications of the model used. This underscores the complexity of decision making in older adults and the important role of a comprehensive risk assessment and evaluation of patient preferences and priorities.

Even when adequate risk stratification tools become available, efforts must still be made to establish the appropriate treatment thresholds in older patients. Current guidelines recommend consideration of statin therapy in intermediate risk patients at a risk threshold of 7.5\% 10-year risk, ${ }^{20,}{ }^{28}$ with some suggesting a treatment benefit with an estimated 10-year risk as low as $5 \% .^{29}$ Older adults ( $\geq 75$ years old) universally meet the $7.5 \% 10$-year treatment threshold identified as the lower limit of "intermediate risk" for younger patients; in fact, the majority of individuals $\geq 75$ years old had a 5 -year risk of 
7.5\%. Identifying the optimal treatment threshold for older patients represents a critical prevention challenge. Saeed et al. recently suggested a timeframe for risk assessment of 35 years for individuals $\geq 75$ years, as well as a greater emphasis on heart failure events rather than the ASCVD events predicted by the PCE. ${ }^{17}$ We agree that a shorter timeframe for risk assessment of $\sim 5$ years provides the most useful information to inform risk-benefit conversations with older adult patients. A patient-centered discussion should guide primary prevention statin treatment decisions in all patients, but takes on even greater importance among individuals $\geq 75$ years old who often present with a complex collection of competing risks paired with a less robust evidence base supporting benefit.

This study has several limitations. First, the study population was limited to patients in the pooled cohorts for whom we had access to the necessary variables to calculate the PCE; therefore, the resulting cohort may be somewhat different from the United States older adult population at large and may overestimate performance of the PCE when used in routine clinical practice. Second, because the PCE were derived using data from similar cohorts used in this study (but different exams), this analysis likely overestimates the performance of the PCE. Third, we utilized pooled cohort data from the 1990s and early $2000 \mathrm{~s}$. With the improvement of preventive therapies over the last decade, the observed event rates may be improved in a more contemporary population. Finally, our study calculated event rates at 5 years, which may not be familiar to clinicians who are more comfortable working with 10-year predicted risk; however, 5-year event rates are more likely to represent a meaningful timeframe for older adults with a comparatively modest life expectancy.

In conclusion, the PCE performs poorly among adults 75 years of age and older, demonstrating both poor discrimination and calibration. New risk stratification tools, developed and validated in older populations using more geriatricspecific risk modifiers are necessary to more effectively discriminate risk and better inform preventive care decisions.

Acknowledgments: We thank Erin Campbell, MS, for her editorial contributions to this manuscript. Ms. Campbell did not receive compensation for her contributions, apart from her employment at the institution where this study was conducted.

Corresponding Author: Michael G. Nanna, MD; Duke Clinical Research Institute Duke University School of Medicine, Durham, NC 27710, USA (e-mail: michael.nanna@duke.edu).

Funding Source Dr. Navar is funded by NIH K01HL133416-01. Dr. Nanna is supported by NIH training grant T-32-HLO69749-15.

\section{Compliance with Ethical Standards:}

Conflict of Interest: MG Nanna: No relationship(s) to disclose. ED Peterson: Research Grant: Significant; Amgen, Sanofi, Astrazeneca, Merck. Consultant/Advisory Board; Modest; Amgen. Consultant/ Advisory Board: Significant; AstraZeneca, Merck, and Sanofi Aventis. $D$ Wojdyla: No relationship(s) to disclose.
AM Navar: Research Grant: Significant; Amarin, Janssen, Amgen, Sanofi, and Regeneron Pharmaceuticals. Consultant/Advisory Board: Significant; Amarin, Amgen, Novonordisk, AstraZeneca, Sanofi and Regeneron.

\section{REFERENCES}

1. Roberts AW, Ogunwole SU, Blakeslee L, Rabe MA. The population 65 years and older in the United States: 2016. American Community Survey Reports, ACS-38. U.S. Census web site. https://www.census. gov/content/dam/Census/library/publications/2018/acs/ACS-38.pdf. Published October 2018. Accessed April 1, 2019.

2. Heron M. Deaths: leading causes for 2016. Natl Vital Stat Rep. 2018;67:1-76.

3. Goff DC Jr., Lloyd-Jones DM, Bennett G, Coady S, D'Agostino RB, Gibbons R, et al. 2013 ACC/AHA guideline on the assessment of cardiovascular risk: a report of the American College of Cardiology/ American Heart Association Task Force on Practice Guidelines. Circulation. 2014;129:S49-S73.

4. Navar-Boggan AM, Peterson ED, D'Agostino RB Sr, Pencina MJ, Sniderman AD. Using age- and sex-specific risk thresholds to guide statin therapy: one size may not fit all. J Am Coll Cardiol. 2015;65:1633-

5. Cook NR, Ridker PM. Calibration of the Pooled Cohort Equations for Atherosclerotic Cardiovascular Disease: An Update. Ann Intern Med. 2016;165:786-794.

6. Stone NJ, Robinson JG, Lichtenstein AH, Bairey Merz CN, Blum CB, Eckel RH, et al. 2013 ACC/AHA guideline on the treatment of blood cholesterol to reduce atherosclerotic cardiovascular risk in adults: a report of the American College of Cardiology/American Heart Association Task Force on Practice Guidelines. J Am Coll Cardiol. 2014;63:2889-934.

7. Rana JS, Tabada GH, Solomon MD, Lo JC, Jaffe MG, Sung SH, et al. Accuracy of the atherosclerotic cardiovascular risk equation in a large contemporary, multiethnic population. J Am Coll Cardiol. 2016;67:211830.

8. Muntner P, Colantonio LD, Cushman M, Goff DC Jr., Howard G, Howard VJ, et al. Validation of the atherosclerotic cardiovascular disease Pooled Cohort risk equations. JAMA. 2014;311:1406-15.

9. Demler OV, Payter NP, Cook NR. Tests of calibration and goodness of fit in the survival setting. Statist. Med. 2015;34:1659-1680.

10. Appelman Y, van Rijn BB, Ten Haaf ME, Boersma E, Peters SA. Sex differences in cardiovascular risk factors and disease prevention. Atherosclerosis. 2015;241:211-8.11.

11. Mosca L, Barrett-Connor E, Wenger NK. Sex/gender differences in cardiovascular disease prevention: what a difference a decade makes. Circulation. 2011;124:2145-54.

12. Yadlowsky S, Hayward RA, Sussman JB, McClelland RL, Min YI, Basu S. Clinical implications of revised Pooled Cohort Equations for estimating atherosclerotic cardiovascular disease risk. Ann Intern Med. 2018;169:20-9.

13. DeFilippis AP, Young R, Carrubba CJ, McEvoy JW, Budoff MJ, Blumenthal RS, et al. An analysis of calibration and discrimination among multiple cardiovascular risk scores in a modern multiethnic cohort. Ann Intern Med. 2015;162:266-75.

14. Ridker PM, Cook NR. Statins: new American guidelines for prevention of cardiovascular disease. Lancet. 2013;382:1762-5.

15. Andersson C, Enserro D, Larson MG, Xanthakis V, Vasan RS. Implications of the US cholesterol guidelines on eligibility for statin therapy in the community: comparison of observed and predicted risks in the Framingham Heart Study Offspring Cohort. J Am Heart Assoc. 2015;4:1-10.

16. DeFilippis AP, Young R, McEvoy JW, Michos ED, Sandfort V, Kronmal RA, et al. Risk score overestimation: the impact of individual cardiovascular risk factors and preventive therapies on the performance of the American Heart Association-American College of CardiologyAtherosclerotic Cardiovascular Disease risk score in a modern multiethnic cohort. Eur Heart J. 2017;38:598-608.

17. Saeed A, Nambi V, Sun W, Virani SS, Taffet GE, Deswal A, et al. Shortterm global cardiovascular disease risk prediction in older adults. J Am Coll Cardiol. 2018;71:2527-36.

18. Forman DE, Rich MW, Alexander KP, Zieman S, Maurer MS, Najjar SS, et al. Cardiac care for older adults. Time for a new paradigm. J Am Coll Cardiol. 2011;57:1801-10.

19. Nanna MG, Navar AM, Wang TY, Mi X, Virani SS, Louie MJ, et al. Statin use and adverse effects among adults $>75$ years of age: insights 
from the Patient and Provider Assessment of Lipid Management (PALM) Registry. J Am Heart Assoc. 2018;7:1-9.

20. Grundy SM, Stone NJ, Bailey AL, Beam C, Birtcher KK, Blumenthal RS, et al. 2018 AHA/ACC/AACVPR/AAPA/ABC/ACPM/ADA/AGS/ APhA/ASPC/NLA/PCNA Guideline on the Management of Blood Cholesterol: a report of the American College of Cardiology/American Heart Association Task Force on Clinical Practice Guidelines. J Am Coll Cardiol. 2019;73:e285-350.

21. McClelland RL, Jorgensen NW, Budoff M, Blaha MJ, Post WS, Kronmal RA, et al. 10-year coronary heart disease risk prediction using coronary artery calcium and traditional risk factors: derivation in the MESA (Multi-Ethnic Study of Atherosclerosis) with validation in the HNR (Heinz Nixdorf Recall) Study and the DHS (Dallas Heart Study). J Am Coll Cardiol. 2015;66:1643-53.

22. Schmermund A, Mohlenkamp S, Stang A, Gronemeyer D, Seibel R, Hirche $\mathbf{H}$, et al. Assessment of clinically silent atherosclerotic disease and established and novel risk factors for predicting myocardial infarction and cardiac death in healthy middle-aged subjects: rationale and design of the Heinz Nixdorf RECALL Study. Risk factors, evaluation of coronary calcium and lifestyle. Am Heart J. 2002;144:212-8.

23. Victor RG, Haley RW, Willett DL, Peshock RM, Vaeth PC, Leonard D, et al. The Dallas Heart Study: a population-based probability sample for the multidisciplinary study of ethnic differences in cardiovascular health. Am J Cardiol. 2004;93:1473-80.
24. Karmali KN, Goff DC Jr., Ning H, Lloyd-Jones DM. A systematic examination of the 2013 ACC/AHA pooled cohort risk assessment tool for atherosclerotic cardiovascular disease. J Am Coll Cardiol. 2014;64:95968.

25. Garcia M, Mulvagh SL, Merz CN, Buring JE, Manson JE. Cardiovascular disease in women: clinical perspectives. Circ Res. 2016;118:127393.

26. Pencina MJ, D'Agostino RB Sr, Larson MG, Massaro JM, Vasan RS. Predicting the 30-year risk of cardiovascular disease: the Framingham Heart Study. Circulation. 2009;119:3078-84.

27. Van Kempen BJ, Ferket BS, Kavousi M, Leening MJ, Steyerberg EW, Ikram MA, et al. Performance of Framingham cardiovascular disease (CVD) predictions in the Rotterdam Study taking into account competing risks and disentangling CVD into coronary heart disease (CHD) and stroke. Int J Cardiol. 2014;171:413-8.

28. Arnett DK, Blumenthal RS, Albert MA, Buroker AB, Goldberger ZD, Hahn EJ, et al. 2019 ACC/AHA Guideline on the Primary Prevention of Cardiovascular Disease: a report of the American College of Cardiology/ American Heart Association Task Force on Clinical Practice Guidelines. J Am Coll Cardiol. 2019;74:e177-e232.

29. Cholesterol Treatment Trialists' (CTT) Collaborators, Mihaylova B, Emberson J, Blackwell L, Keech A, Simes J, et al. The effects of lowering LDL cholesterol with statin therapy in people at low risk of vascular disease: meta-analysis of individual data from 27 randomized trials. Lancet. 2012;380:581-90.

Publisher's Note Springer Nature remains neutral with regard to jurisdictional claims in published maps and institutional affiliations. 